\title{
DESCRIBING IDEALS OF ENDOMORPHISM RINGS
}

\author{
Brendan Goldsmith and Simone Pabst
}

\section{Introduction}

It is well known that the ring of linear transformations of a finite dimensional vector space is simple, i.e. it has no non-trivial proper two-sided ideals. It is, perhaps, not so well known that the (twosided) ideals in the ring of linear transformations of an infinite dimensional vector space can be characterized by a single cardinal invariant, [1]. It is therefore reasonably natural to ask if there is a generalization of Baer's result to ideals in the endomorphism ring of a wider class of modules. The purpose of this present work is to explore this possibility.

A first generalization is to replace the underlying field of the vector space by a ring. A natural extension of the concept of a field is a discrete valuation ring since a discrete valuation ring modulo its Jacobson radical is a field. Recall (see e.g. [5]) that $R$ is a discrete valuation ring if $R$ is a principal ideal ring with exactly one maximal ideal or, alternatively, with one prime element $p$. In particular the ring of $p$-adic rationals, $\mathbf{Z}_{p}$, is a discrete valuation ring and the reader may replace all references to discrete valuation rings with the $p$-adic rationals without any serious loss in generality. Also, recall that if $E$ is a ring then the Jacobson radical of $E$ is defined to be the intersection of all maximal ideals of $E$. It is well known that this is equivalent to the set of all elements $x \in E$ such that $r x s$ is quasi-regular for all $r$ and $s$ in $E$, i.e. those elements $x$ for which $1-r x s$ is a unit.

Since a vector space over a field $F$ is a free $F$-module, an obvious question to ask is whether a corresponding characterization of ideals in the endomorphism ring of a free module over a 
discrete valuation ring exists. We address this problem in $\S 3$ and obtain Baer's Theorem for vector spaces as a corollary to our main result Theorem 3.3.

Whilst free modules over a discrete valuation ring are an obvious generalization of vector spaces it is, perhaps, not so obvious that complete modules over a complete discrete valuation ring $R$ have many similar properties to vector spaces (cf. [4]). Recall that a complete discrete valuation ring is a discrete valuation ring which is complete in its $p$-adic topology (i.e. the linear topology with basis of neighbourhoods of 0 given by $p^{n} R, n \geq 0$ ), where $p$ is the only prime element of $R$. In $\S 4$ we discuss the ideal structure of the endomorphism ring of such modules and achieve a complete characterization modulo the Jacobson radical.

We conclude this introduction by reviewing a number of standard concepts in module/abelian group theory. If $R$ is discrete valuation ring with prime element $p$ then we say that an $R$ submodule $H$ of the $R$-module $G$ is pure in $G$ if $H \cap p^{n} G=p^{n} H$ for all $n \geq 0$. If we consider the module $G$ as a topological module furnished with the $p$-adic topology (i.e. a basis of neighbourhoods of 0 is given by $p^{n} G, n \geq 0$ ), then $H$ is pure in $G$ precisely if the $p$-adic topology on $H$ coincides with the induced subspace topology. Also an $R$-module $D$ is divisible if given any $d \in D$ we can solve the equation $p^{n} x=d$ in $D$; a module is reduced if it has no non-trivial divisible submodules. (It is well known that a torsion-free divisible $R$-module is a direct sum of copies of the quotient field $Q$ of $R$.) Notice that if $H \subseteq G$ then $G / H$ divisible is equivalent to $H$ being dense in the $p$-adic topology on $G$.

Finally note that maps are written on the right and the word ideal will always mean a two-sided ideal.

\section{Preliminaries}

In this section we state a few well-known results on modules over complete discrete valuation rings. Throughout the section $R$ shall be a complete discrete valuation ring with prime element $p$. In considering torsion-free $R$-modules, the concept of a basic submodule is useful; a submodule $B$ of a torsion-free $R$-module $A$ is called a basic submodule of $A$ if $B$ is a free $R$-module such that 
$A / B$ is divisible and $B$ is pure in $A$.

The first lemma is due to R. B. Warfield, [8]; it tells us how to obtain a basic submodule from the $R / p R$-vector space $A / p A$.

Lemma 2.1 Let $A$ be a torsion-free $R$-module and $\pi: A \longrightarrow$ $A / p A$ the natural epimorphism. Let $\left\{x_{i} \mid i \in I\right\}$ be an $R / p R-$ basis of $A / p A$ and choose $y_{i} \in A(i \in I)$ such that $y_{i} \pi=x_{i}$. Then the submodule $B$ generated by $\left\{y_{i} \mid i \in I\right\}$ is a basic submodule of $A$. Moreover every basic submodule of $A$ arises in this way.

The lemma ensures the existence of a basic submodule $B$ and the uniqueness of its $\operatorname{rank} \operatorname{rk}(B)$ where $\operatorname{rk}(B)$ is the usual rank of a free $R$-module. Hence we may define the rank of a torsion-free $R-$ module $A$ as the rank of its basic submodule, i.e. $\operatorname{rk}(A)=\operatorname{rk}(B)$. Since the divisibility of the quotient module $A / B$ is equivalent to the density of $B$ in $A$ in the $p$-adic topology we obtain the following well-known characterization of complete reduced torsion-free $R-$ modules; (2) is essentially due to I. Kaplansky, [5], and (3) is similar to a result proved for $p$-groups by H. Leptin, [6].

Proposition 2.2 The following properties of a reduced torsionfree $R$-module $A$ are equivalent:

(1) $A$ is complete.

(2) If $B$ is a basic submodule of $A$, then $A$ is the completion of $B$.

(3) If $B$ is a basic submodule of $A$, then every $R$-homomorphism $B \longrightarrow A$ extends uniquely to an $R$-endomorphism of $A$.

Next we state a few facts on complete reduced torsion-free $R$-modules; the proofs can be found in [2], [5], and [7].

Lemma 2.3 Let $A$ and $A^{\prime}$ be complete reduced torsion-free $R_{-}^{-}$ modules.

(1) If $\theta$ is an endomorphism of $A$, then both $\operatorname{ker} \theta$ and $\operatorname{im} \theta$ are complete.

(2) If $S$ is a pure submodule of $A$ and is complete, then $S$ is a direct summand of $A$.

(3) $A$ is isomorphic to $A^{\prime}$ if and only if $\operatorname{rk}(A)=\operatorname{rk}\left(A^{\prime}\right)$.

We finish this section with a standard result on the Jacobson radical of the endomorphism ring of a complete reduced torsionfree $R$-module; a proof may be found in [7]. 
Proposition 2.4 Let $E$ denote the endomorphism ring $\operatorname{End}(A)$ of the complete reduced torsion-free $R$-module $A$. Then

(1) $J(E)=p E=E p$;

(2) $E / J(E) \cong \operatorname{End}_{R / p R}(A / p A)$;

(3) $J(E)=\{\phi \in E \mid A \phi \subseteq p A\}$.

\section{Free modules over discrete valuation rings}

Here we shall discuss ideals of endomorphism rings of free $R-$ modules over discrete valuation rings $R$. We will deduce Baer's Theorem on vector spaces as a corollary to our main result. Before we restrict our attention to modules over discrete valuation rings we prove a result (Proposition 3.2) which is true for modules in general. The definition of a direct endomorphism will be useful. An endomorphism $\mu$ of $A$ is called $k$-direct [im-direct $]$ if $\operatorname{ker}(\mu)$ $[\operatorname{Im}(\mu)]$ is a direct summand of $A$, and $\mu$ is said to be a direct endomorphism if it is both k-direct and im-direct. First we need:

Lemma 3.1 If $\sigma$ is a direct endomorphism of $A$, then there exists an endomorphism $\eta$ of $A$ such that

(a) $\sigma \eta$ and $\eta \sigma$ are both idempotent, and

(b) $\operatorname{Im}(\sigma)=\operatorname{Im}(\eta \sigma), \operatorname{Im}(\sigma \eta)=\operatorname{Im}(\eta), \operatorname{ker}(\eta)=\operatorname{ker}(\eta \sigma)$, and $\operatorname{ker}(\sigma \eta)=\operatorname{ker}(\sigma)$.

Proof: By our assumption, we may write

$$
A=\operatorname{Im}(\sigma) \oplus S \text { and } A=\operatorname{ker} \sigma) \oplus T .
$$

Then the restriction $\sigma_{T}: T \longrightarrow \operatorname{Im}(\sigma)$ is an isomorphism. Hence there exists $\tau: \operatorname{Im}(\sigma) \longrightarrow T$ such that

$$
\tau\left(\sigma_{T}\right)=\mathrm{id}_{\operatorname{Im}(\sigma)} \text { and }\left(\sigma_{T}\right) \tau=\mathrm{id}_{T} .
$$

Now let $\eta: A \longrightarrow A$ be defined by

$$
\eta_{\operatorname{Im}(\sigma)}=\tau \text { and } \operatorname{ker} \eta=S .
$$

We shall see that $\eta$ is the required endomorphism.

First we show that $\sigma \eta$ and $\eta \sigma$ are idempotent. Let $x$ be an arbitrary element of $A$. Then

$$
x(\sigma \eta)^{2}=x \sigma \eta \sigma \eta=x \sigma \tau \sigma \eta=x \sigma \eta,
$$


since $\tau \sigma$ is the identity on $\operatorname{Im}(\sigma)$. So $(\sigma \eta)^{2}=\sigma \eta$. Now let $x=$ $a \sigma+s \in A$, where $a \in A$ and $s \in S$. Then

$$
x(\eta \sigma)^{2}=x \eta \sigma \eta \sigma=a \sigma \eta \sigma \eta \sigma=(a \sigma) \eta \sigma=x \eta \sigma .
$$

Thus, $(\eta \sigma)^{2}=\eta \sigma$, as required.

To prove part (b) we make the following calculations:

$$
\begin{aligned}
\underline{\operatorname{Im}(\eta \sigma)} & =A \eta \sigma=A \sigma \eta \sigma=A \sigma \tau \sigma=A \sigma=\underline{\operatorname{Im}(\sigma) ;} \\
\underline{\operatorname{Im}(\sigma \eta)} & =A \sigma \eta=A \sigma \tau=T=\underline{\operatorname{Im}(\eta) ;} \\
\underline{\operatorname{ker}(\eta \sigma)} & =\{x \in A \mid x \eta \sigma=0\}=\{x \in A \mid x \eta \in \operatorname{ker}(\sigma)\} \\
& =\{x \in A \mid x \eta \in \operatorname{ker}(\sigma) \cap T=0\}=\underline{\operatorname{ker}(\sigma) ;} \\
\underline{\operatorname{ker}(\sigma \eta)} & =\{x \in A \mid x \sigma \eta=0\}=\{x \in A \mid x \sigma \tau=0\} \\
& =\{x \in A \mid x \sigma=0\}=\underline{\operatorname{ker}(\sigma) .} .
\end{aligned}
$$

This completes the proof.

Proposition 3.2 Let $I$ be an ideal of the endomorphism ring $\operatorname{End}(A)$ of $A$ such that all $\mu \in I$ are $k$-direct. Moreover assume that I contains a direct endomorphism $\sigma$. If $\alpha$ is an im-direct endomorphism of $A$ such that $\operatorname{Im}(\alpha)$ is isomorphic to a direct summand of $\operatorname{Im}(\sigma)$, then $\alpha$ belongs to the ideal $I$.

Proof: Let $I, \sigma$, and $\alpha$ be as above. Then we can write

$$
A=\operatorname{Im}(\sigma) \oplus S=\operatorname{Im}(\alpha) \oplus T \text { and } \operatorname{Im}(\sigma)=R \oplus C,
$$

where $R \cong_{\phi^{\prime}} \operatorname{Im}(\alpha)$. We extend $\phi^{\prime}$ to an endomorphism $\phi$ of $A$ by

$$
\phi_{R}=\phi^{\prime} \text { and } \phi_{C \oplus S}=0 .
$$

Now consider the endomorphism $\sigma \phi \in I$. Since $\sigma \phi$ belongs to $I$ it is $\mathrm{k}$-direct. Moreover,

$$
A \sigma \phi=(R \oplus C) \phi=R \phi=R \phi^{\prime}=\operatorname{Im}(\alpha),
$$

which is a direct summand of $A$. Hence $\sigma \phi$ is direct and we may apply Lemma 3.1 to $\sigma \phi$. Thus there exists $\eta \in \operatorname{End}(A)$ such that 
$\eta \sigma \phi$ is an idempotent and $\operatorname{Im}(\eta \sigma \phi)=\operatorname{Im}(\sigma \phi)=\operatorname{Im}(\alpha)$. Therefore, for any $x \in A$, there is $y \in A$ with $x \alpha=y \eta \sigma \phi$. Hence,

$$
(x \alpha) \eta \sigma \phi=y(\eta \sigma \phi)^{2}=y \eta \sigma \phi=x \alpha,
$$

which implies that $\alpha=\alpha \eta \sigma \phi$ is in $I$, since $I$ is an ideal.

Now let $R$ be a discrete valuation ring and $A$ a free $R$-module. The next theorem tells us something about ideals $I$ containing a direct endomorphism of a given rank. Recall that the rank of an endomorphism $\sigma$ is defined to be the rank of the free $R$-module $\operatorname{Im}(\sigma)$.

Theorem 3.3 Let I be an ideal of $\operatorname{End}(A)$. If I contains a direct endomorphism of rank $\kappa$, then I contains all endomorphisms of rank less than or equal to $\kappa$.

Proof: First we show that all endomorphisms of $A$ are k-direct. Let $\mu$ be any endomorphism of $A$. Then $A / \operatorname{ker}(\mu) \cong \operatorname{Im}(\mu)$, where $\operatorname{Im}(\mu)$ is free and hence projective. Thus there exists a homomorphism $\phi: \operatorname{Im}(\mu) \longrightarrow A$ with $\phi \mu=\operatorname{id}_{A}$. We show that $A=\operatorname{ker}(\mu) \oplus(\operatorname{Im}(\mu)) \phi$. Let $x \in \operatorname{ker}(\mu) \cap(\operatorname{Im}(\mu)) \phi$. Then there is $y \in A$ such that $x=y \mu \phi$ and

$$
0=x \mu=y \mu \phi \mu=y \mu,
$$

since $\phi \mu=\operatorname{id}_{A}$, and thus $x=y \mu \phi=0 \phi=0$. Also,

$$
a=a \mu \phi+(a-a \mu \phi), \text { with }(a-a \mu \phi) \mu=a \mu-a \mu \phi \mu=0
$$

for any $a \in A$, again since $\phi \mu=\mathrm{id}_{A}$. Thus $\operatorname{ker}(\mu)$ is a direct summand of $A$, implying that $\mu$ is $\mathrm{k}$-direct.

Now let $\sigma \in I$ be a direct endomorphism of $A$ of rank $\kappa$, i.e. $A=\operatorname{Im}(\sigma) \oplus S$ and $\operatorname{rk}(\operatorname{Im}(\sigma))=\kappa$. Next we prove that all direct (im-direct) endomorphisms $\alpha$ with $\operatorname{rk}(\alpha) \leq \kappa$ are elements of $I$. Let $\alpha$ be such an endomorphism. Then

$$
A=\operatorname{Im}(\alpha) \oplus T \text { and } \operatorname{rk}(\alpha)=\operatorname{rk}(\operatorname{Im}(\alpha)) \leq \kappa .
$$

Since $\operatorname{Im}(\alpha)$ and $\operatorname{Im}(\sigma)$ are free $R$-modules with $\operatorname{rk}(\operatorname{Im}(\alpha)) \leq$ $\operatorname{rk}(\operatorname{Im}(\sigma))$, there exists a direct summand of $\operatorname{Im}(\sigma)$ which is isomorphic to $\operatorname{Im}(\alpha)$. Thus we may apply Proposition 3.2, which implies that $\alpha \in I$. 
Finally let $\phi$ be any endomorphism of $A$ with $\operatorname{rk}(\phi) \leq \kappa$. Since $\phi$ is k-direct, we may express $A$ as $A=\operatorname{ker}(\phi) \oplus C$, where $C \cong A / \operatorname{ker}(\phi) \cong \operatorname{Im}(\phi)$. Thus

$$
\operatorname{rk}(C)=\operatorname{rk}(\phi) \leq \kappa .
$$

Let $\pi$ be the projection of $A$ onto $C$ with $\operatorname{ker}(\pi)=\operatorname{ker}(\phi)$. Obviously $\pi$ is a direct endomorphism with $\operatorname{rk}(\pi)=\operatorname{rk}(C) \leq \kappa$. Thus $\pi \in I$. Hence $\phi=\pi \phi$ is an element of $I$ and this completes proof.

Note that the previous theorem holds for free modules over any ring $R$ having the property that submodules of free modules are free; e.g. all principal ideal domains have this property. So, in particular, Theorem 3.3 holds for a field. In this case we get even more, namely, we can characterize the ideals of the endomorphism ring $\operatorname{End}(A)$ of a vector space $A$.

Corollary 3.4 Let $A$ be a vector space over a field $R$. Then the only ideals of $\operatorname{End}(A)$ are the ideals $E_{\kappa}\left(\kappa \geq \aleph_{0}\right)$ defined by $E_{\kappa}=\{\alpha \in \operatorname{End}(A) \mid \operatorname{rk}(\alpha)<\kappa\}$.

Proof: Note first that all endomorphisms of a vector space $A$ are direct. Hence, in this case Theorem 3.3 reads as:

If $\sigma$ is an element of an ideal I, then I contains every endomorphism $\alpha$ with $\operatorname{rk}(\alpha) \leq \operatorname{rk}(\sigma)$.

It is easy to check that, for each $\kappa \geq \aleph_{0}, E_{\kappa}$ is an ideal of $\operatorname{End}(A)$. Write $E_{0}$ for $E_{\aleph_{0}}$, the ideal of all finite rank endomorphisms.

Now, let $I \neq 0$ be an arbitrary ideal which is properly contained in $\operatorname{End}(A)$. Since $I$ is non-trivial, there exists a non-zero endomorphism $\sigma \in I$. If $\sigma$ is of infinite rank then, obviously, $E_{0} \subseteq I$. So suppose $\sigma$ is of finite rank $n \geq 1$. In this case, $I$ contains all endomorphisms of rank less than or equal to $n$. Thus if $e$ is an element of a given basis $B$, then $I$ contains the projection $\pi_{e}$ onto the one-dimensional subspace generated by $e$ along the subspace generated by the remaining basis elements. Therefore all finite sums $\sum_{i=1}^{k} \pi_{e_{i}}\left(e_{i} \in B\right)$ of such projections belong to $I$ and hence, for any $k \in \mathbf{N}$, there is an endomorphism of rank $k$ belonging to $I$. This implies that all endomorphisms of finite rank are contained in the ideal $I$ and so in either case we deduce that 
$E_{0} \subseteq I$. Moreover $E_{\kappa+1} \subseteq I$ whenever $\eta \in I$ for some $\eta$ of rank $\kappa$. Let $\tau+1$ be the smallest cardinal with $E_{\tau+1} \nsubseteq I$ (we may consider a successor cardinal since the ideals $E_{\kappa}$ form a smooth increasing chain). Then all $\eta \in I$ have rank less than $\tau$ and hence $I \subseteq E_{\tau}$. Also $E_{\tau} \subseteq I$ by the minimality of $\tau+1$, thus $I=E_{\tau}$.

\section{Complete modules over complete discrete valuation} rings

In the last section we turn our attention to complete reduced torsion-free $R$-modules $A$ over complete discrete valuation rings $R$. Recall that the rank of a reduced torsion-free $R$-module over a complete discrete valuation ring is the rank of a basic submodule $B$ of $A$ (see Section 2). Again we define the rank of an endomorphism as the rank of its image. Moreover we call an endomorphism $\alpha$ of $A$ a pure endomorphism if $\operatorname{Im}(\alpha)$ is a pure submodule of $A$.

First we present a result which is similar to Theorem 3.3: for a complete reduced torsion-free $R$-module $A$ over a complete discrete valuation ring $R$ we can prove

Theorem 4.1 Let $I$ be an ideal of $\operatorname{End}(A)$. If I contains a pure endomorphism $\sigma$ of rank $\kappa$ then I contains all endomorphisms $\alpha$ with $\operatorname{rk}(\alpha) \leq \kappa$.

Proof: Firstly we show that any endomorphism $\mu$ of $A$ is k-direct. By Lemma 2.3 it suffices to show that $\operatorname{ker}(\mu)$ is pure in $A$ for any $\mu \in \operatorname{End}(A)$. If $x=p^{n} a$ with $x \in \operatorname{ker} \mu$ and $a \in A$, we have $\left(p^{n} a\right) \mu=x \mu=0$. Hence $p^{n}(a \mu)=0$, which implies $a \mu=0$ since $A$ is torsion-free. So,

$$
p^{n} A \cap \operatorname{ker}(\mu)=p^{n} \operatorname{ker}(\mu),
$$

that is, $\operatorname{ker}(\mu)$ is pure in $A$ and thus $\mu$ is $\mathrm{k}$-direct for any $\mu \epsilon$ $\operatorname{End}(A)$.

Let $\sigma$ be a pure endomorphism in $I$ and assume first that $\alpha$ is a pure endomorphism of $A$. Then, by Lemma 2.3 , both $\operatorname{Im}(\sigma)$ and $\operatorname{Im}(\alpha)$ are direct summands of $A$, i.e. we may write $A$ as

$$
A=\operatorname{Im}(\sigma) \oplus S=\operatorname{Im}(\alpha) \oplus T
$$


If $B_{\alpha}, B_{\sigma}$ are basic submodules of $\operatorname{Im}(\alpha)$ and $\operatorname{Im}(\sigma)$ respectively, then there exists a direct summand $D$ of $B_{\sigma}$ of rank

$$
\operatorname{rk}(\alpha)=\operatorname{rk}\left(B_{\alpha}\right) \leq \operatorname{rk}\left(B_{\sigma}\right)=\operatorname{rk}(\sigma),
$$

which is isomorphic to $B_{\alpha}$. We may extend this isomorphism to an isomorphism of the completions $\operatorname{Im}(\alpha)$ and $\widehat{D}$ of $B_{\alpha}$ and $D$ respectively. Moreover, since $D$ is pure in $B_{\sigma}$, the completion $\widehat{D}$ is pure in $\operatorname{Im}(\sigma)$, hence $\widehat{D}$ is a direct summand of $\operatorname{Im}(\sigma)$ which is isomorphic to $\operatorname{Im}(\alpha)$. Thus we may apply Proposition 3.2 which implies that $\alpha \in I$. We have shown that all pure endomorphisms $\alpha$ with $\operatorname{rk}(\alpha) \leq \operatorname{rk}(\sigma)$ are contained in $I$. So, in particular, all idempotents $\pi$ with $\operatorname{rk}(\pi) \leq \operatorname{rk}(\sigma)$ belong to $I$.

Finally, let $\phi$ be any endomorphism of $A$ with $\operatorname{rk}(\phi) \leq \operatorname{rk}(\sigma)$. Then $A=\operatorname{ker}(\phi) \oplus C$, where $C \cong A / \operatorname{ker}(\phi) \cong \operatorname{Im}(\phi)$, and so $\operatorname{rk}(C)=\operatorname{rk}(\alpha)$. If $\pi$ denotes the projection onto $C$ with $\operatorname{ker}(\pi)=$ $\operatorname{ker}(\phi)$ then $\pi \in I$ since

$$
\operatorname{rk}(\pi)=\operatorname{rk}(C)=\operatorname{rk}(\phi) \leq \operatorname{rk}(\sigma) .
$$

Therefore $\phi=\pi \phi$ is an element of $I$.

The previous theorem, however, does not characterize the ideals of $\operatorname{End}(A)$ since there are ideals which do not contain a pure endomorphism, for example, $p \operatorname{End}(A)$. Instead of using similar arguments as in the case of free $R$-modules we shall now use Corollary 3.4 on vector spaces to determine the ideals $I$ of $\operatorname{End}(A)$ modulo their Jacobson radicals. First we consider the Jacobson radicals $J\left(E_{\kappa}\right)$ of the ideals $E_{\kappa}$ where

$$
E_{\kappa}=\{\eta \in \operatorname{End}(A) \mid \operatorname{rk}(\eta)<\kappa\}
$$

for $\kappa \geq \aleph_{0}$.

Lemma 4.2 Let $E_{\kappa}$ be an ideal as defined above. Then the Jacobson radical $J\left(E_{\kappa}\right)$ coincides with the ideal $p E_{\kappa}$.

Proof: First we show that $p E_{\kappa}=E_{\kappa} \cap p \operatorname{End}(A)$. Let $p \alpha \in E_{\kappa}$ with $\alpha \in \operatorname{End}(A)$. Then $A=A_{1} \oplus \operatorname{ker}(p \alpha)$ and $\operatorname{rk}\left(A_{1}\right)<\kappa$. Since $A$ is torsion-free, $\operatorname{ker}(p \alpha)=\operatorname{ker}(\alpha)$. Hence $A \alpha=A_{1} \alpha$ and $\operatorname{rk}(\alpha)<\kappa$. 
Thus $\alpha \in E_{\kappa}$. By Proposition 2.4, $p \operatorname{End}(A)=J(\operatorname{End}(A))$ and so it follows that

$$
E_{\kappa} \cap p \operatorname{End}(A)=E_{\kappa} \cap J(\operatorname{End}(A)) .
$$

But $E_{\kappa} \cap J(\operatorname{End}(A))=J\left(E_{\kappa}\right)$ since $E_{\kappa}$ is an ideal of $\operatorname{End}(A)$. Therefore

$$
p E_{\kappa}=E_{\kappa} \cap p \operatorname{End}(A)=E_{\kappa} \cap J(\operatorname{End}(A))=J\left(E_{\kappa}\right) .
$$

This completes the proof.

Next we will show that $E_{\kappa} / J\left(E_{\kappa}\right)$ is isomorphic to a corresponding ideal of the vector space $A / p A$ over the field $R / p R$.

Lemma 4.3 For any cardinal $\kappa \geq \aleph_{0}, E_{\kappa} / J\left(E_{\kappa}\right) \cong E_{\kappa}(A / p A)$.

Proof: Every $\alpha \in E_{\kappa}$ induces an $R / p R$-endomorphism on $A / p A$ since $p A \alpha \subseteq p A$. So we may define a map

$$
\Delta: E_{\kappa} \longrightarrow \operatorname{End}_{R / p R}(A / p A)
$$

by

$$
\alpha \Delta=\bar{\alpha}: A / p A \longrightarrow A / p A \text { with }(a+p A) \bar{\alpha}=a \alpha+p A .
$$

It is easy to check that $\Delta$ is a ring homomorphism. Moreover, the kernel of $\Delta$ is $p E_{\kappa}=J\left(E_{\kappa}\right)$. We show that $\operatorname{Im}(\Delta)=E_{\kappa}(A / p A)$. Certainly, $\operatorname{Im}(\Delta) \subseteq E_{\kappa}(A / p A)$ since the vector space rank of an endomorphism $\bar{\alpha}$ of $A / p A$ cannot be greater than $\operatorname{rk}(\alpha)$. Now let $\eta: A \longrightarrow A / p A$ and $\rho: R \longrightarrow R / p R$ be the endomorphisms defined by

$$
a \eta=a+p A \text { and } r \rho=r+p R .
$$

Let us consider an endomorphism $\phi$ of $A / p A$ of rank less than $\kappa$. We can pick an $R / p R$-basis $\left\{x_{i} \mid i \in I\right\}$ of $A / p A$ such that $x_{i} \phi=0$ for all but less than $\kappa$ of the $x_{i}$. Choose $y_{i} \in A$ such that $y_{i} \eta=x_{i}$ for all $i \in I$. Then the module generated by $\left\{y_{i} \mid i \in I\right\}$ is a basic submodule of $A$ by Lemma 2.1. However

$$
x_{i} \phi=\sum_{i \in I} r_{i j} x_{j}
$$


where $r_{i j} \in R / p R$ with $r_{i j}=0$ for all but finitely many $j$. Choose $s_{i j} \in R$ with $s_{i j} \phi=r_{i j}$ and $s_{i j}=0$ whenever $r_{i j}=0$. Finally, define $\beta: B \longrightarrow B$ by

$$
y_{i} \beta=\sum_{i \in I} s_{i j} y_{j}
$$

so that $y_{i} \beta=0$ for all but less than $\kappa$ of the $y_{i}$. Thus the unique extension of $\beta$ to an endomorphism of $A$ has $\operatorname{rank} \operatorname{rk}(B \beta)<\kappa$ and satisfies $\beta \Delta=\phi$. Hence $\Delta: E_{\kappa} \longrightarrow E_{\kappa}(A / p A)$ is surjective, with $\operatorname{ker} \Delta=p E_{\kappa}=J\left(E_{\kappa}\right)$. Thus $E_{\kappa} / J\left(E_{\kappa}\right) \cong E_{\kappa}(A / p A)$, as required.

We finish the paper with the characterization of the ideals of the endomorphism ring of a complete reduced torsion-free $R$ module $A$ over a complete discrete valuation ring $R$ : modulo their Jacobson radical they are characterized by a single cardinal $\kappa$.

Theorem 4.4 If $I$ is an arbitrary ideal of the endomorphism ring of a complete reduced torsion-free $R$-module $A$, then either $I \subseteq$ $J(\operatorname{End}(A))$ or

$$
I / J(I) \cong E_{\kappa} / J\left(E_{\kappa}\right) \cong E_{\kappa}(A / p A)
$$

for some cardinal $\kappa$.

Proof: Let $I$ be any ideal of $\operatorname{End}(A)$. We consider the mapping $\Delta: I \longrightarrow \operatorname{End}(A / p A)$ defined by

$$
\alpha \Delta=\bar{\alpha} \text { with }(a+p A) \bar{\alpha}=a \alpha+p A .
$$

This defines a ring homomorphism with

$$
\operatorname{ker}(\Delta)=J(I)=I \cap J(\operatorname{End}(A))
$$

which is either equal to $I$ (i.e. $I \subseteq J(\operatorname{End}(A)))$ or is properly contained in $I$. In the latter case $I / J(I) \cong K$ for some nonzero ideal $K$ of $\operatorname{End}(A / p A)$. Thus $K=E_{\kappa}(A / p A)$ for some $\kappa$ by Corollary 3.4. Therefore $I / J(I) \cong E_{\kappa} / J\left(E_{\kappa}\right)$ by Lemma 4.3. 


\section{References}

[1] R. Baer, Linear Algebra and Projective Geometry. Academic Press: New York, 1952.

[2] L. Fuchs, Infinite Abelian Groups, vol. I. Academic Press: New York, 1970.

[3] L. Fuchs, Infinite Abelian Groups, vol. II. Academic Press: New York, 1973.

[4] B. Goldsmith, S. Pabst and A. Scott, Unit sum numbers of rings and modules, to appear.

[5] I. Kaplansky, Infinite Abelian Groups. The University of Michigan, Press, 1962.

[6] H. Leptin, Zur Theorie der überabzählbaren abelschen p-Gruppen, Abh. Math. Sem. Univ. Hamburg 24 (1960), 79-90.

[7] W. Liebert, Characterization of the endomorphism rings of divisible torsion modules and reduced complete torsion-free modules over complete discrete valuation rings, Pacific J. Math. 37 (1971), 141-170.

[8] R. B. Warfield, Homomorphisms and duality for torsion-free groups, Math. Z. 107 (1968), 189-200.

Brendan Goldsmith and Simone Pabst

Department of Mathematics, Statistics and Computer Science, Dublin Institute of Technology,

Kevin Street, Dublin 8. 